\title{
Quantum Transport Properties of Chemically Functionalized Long Semiconducting Carbon Nanotubes
}

\author{
Alejandro Lopez-Bezanilla ${ }^{1}$, Xavier Blase ${ }^{2}$, and Stephan Roche ${ }^{1,3}(\bowtie)$ \\ ${ }^{1}$ Commissariat a l'Energie Atomique, 38054 Grenoble Cedex, France \\ ${ }^{2}$ Institut Néel, CNRS and Université Joseph Fourier, B.P. 166, 38042 Grenoble Cedex 09, France \\ ${ }^{3}$ CIN2 (CSIC-ICN) Barcelona, Campus UAB, E-08193 Bellaterra, Spain
}

Received: 6 January 2010 / Revised: 11 February 2010 / Accepted: 12 February 2010

C The Author(s) 2010. This article is published with open access at Springerlink.com

\begin{abstract}
We present a first-principles study of the electronic transport properties of micrometer long semiconducting carbon nanotubes randomly covered with carbene functional groups. Whereas prior studies suggested that metallic tubes are hardly affected by such addends, we show here that the conductance of semiconducting tubes with standard diameter is, on the contrary, severely damaged. The configurational-averaged conductance as a function of tube diameter, with a coverage of up to one hundred molecules, is extracted. Our results indicate that the search for a conductance-preserving covalent functionalization route remains a challenging issue.
\end{abstract}

\section{KEYWORDS}

Nanotubes, functionalization, carbene, electronic transport

\section{Introduction}

Carbon nanotubes (CNTs) are outstanding materials for exploring quantum physics in low dimensionality. Their unique mechanical, electronic, and transport properties have stimulated a great amount of fundamental and applied research with potential use in various technological devices [1]. In particular, the engineering of sidewall chemical functionalization of CNTs has opened true perspectives in the development of innovative systems such as chemical sensors [2], optically modulated conductors $[3,4]$, or novel switching devices and molecular memories [5-8]. However, many difficulties remain to be overcome in order to benefit from the large diversity of organic chemistry reactions that can be performed on a tube wall together with the exceptional transport properties of pristine CNTs. Indeed, commonly used functionalization processes, such as phenyl group addition, [9] produce a significant number of $\mathrm{sp}^{3}$-like defects which dramatically disrupt the $\pi$-conjugated network of CNTs, severely damaging their electronic conduction ability [10-14]. To circumvent such a problem, [2+1] cycloaddition reactions have been proposed [15-18]. This functionalization is driven by grafted carbene (or nitrene) groups that induce bond cleaving between adjacent sidewall carbon atoms, maintaining the $\mathrm{sp}^{2}$ hybridization and providing sites for further attachment of complex molecules and related functionalities [19]. First-principles calculations have shown that high conductance is preserved in functionalized metallic nanotubes with random coverage of addends [14, 20,21], but the situation for semiconducting nanotubes remains unexplored.

Address correspondence to stephan.roche@cea.fr 
Since standard devices such as field-effect transistors exclusively rely on the use of semiconducting tubes, it is necessary to clarify the effect of functionalization on such systems. In this paper, we report a firstprinciples computational study of the effect of $\mathrm{CH}_{2}$ carbene grafting on the charge transport properties of micrometer long semiconducting CNTs. The evolution of conductance as a function of tube diameter and with random distributions of up to one hundred functional groups is investigated. It is shown that for zigzag tubes, the transition from a quasi-ballistic to a strongly diffusive regime occurs at a much smaller tube diameter when compared to armchair metallic tubes. This is related to a transition from the "open" to "closed" configuration for the nanotube $\mathrm{C}-\mathrm{C}$ bond bridged by the carbene group. As a result, for tube diameters above $1.2-1.4 \mathrm{~nm}$, the conductance of functionalized semiconducting tubes decays rapidly with increasing number of grafted groups, in marked contrast with armchair tubes of equivalent diameter.

\section{Technical details}

The interaction of functional groups with $(n, 0)$ zigzag CNTs ( $n$ stands for the nanotube index) was explored with the SIESTA [22, 23] code, which implements the density functional theory with a linear combination of numerical atomic-like orbital basis sets. Our calculations were performed using the local density approximation for the exchange-correlation potential. Standard norm-conserving pseudopotentials [24] describe the interaction between ionic cores and valence electrons. Split-valence double- $\xi$ polarized basis sets were used in all simulations. Convergency tests showed that such a basis provides well-converged conductance profiles. Interactions between addends and their replica in the periodic one-dimensional cells were avoided by considering six-unit CNT-cells (25.55 $\AA$ ) which guarantee a convergence to pristine $\mathrm{CNT}$ in between the images.

Relaxed atomic positions are obtained when residual atomic forces become smaller than $0.04 \mathrm{eV} / \AA ̊$.

\section{Diameter-dependent functionalization- induced bond cleaving}

In the case of zigzag CNTs, there are two types of $\mathrm{C}-\mathrm{C}$ bond orientations for a grafted $\mathrm{CH}_{2}$ group, namely parallel (II) or skewed $(\backslash)$ with respect to the tube axis. These bond orientations are depicted in Fig. 1 (inset) and Fig. 2 (left inset), respectively, where the bridged tube $\mathrm{C}-\mathrm{C}$ bonds upon carbene adsorption

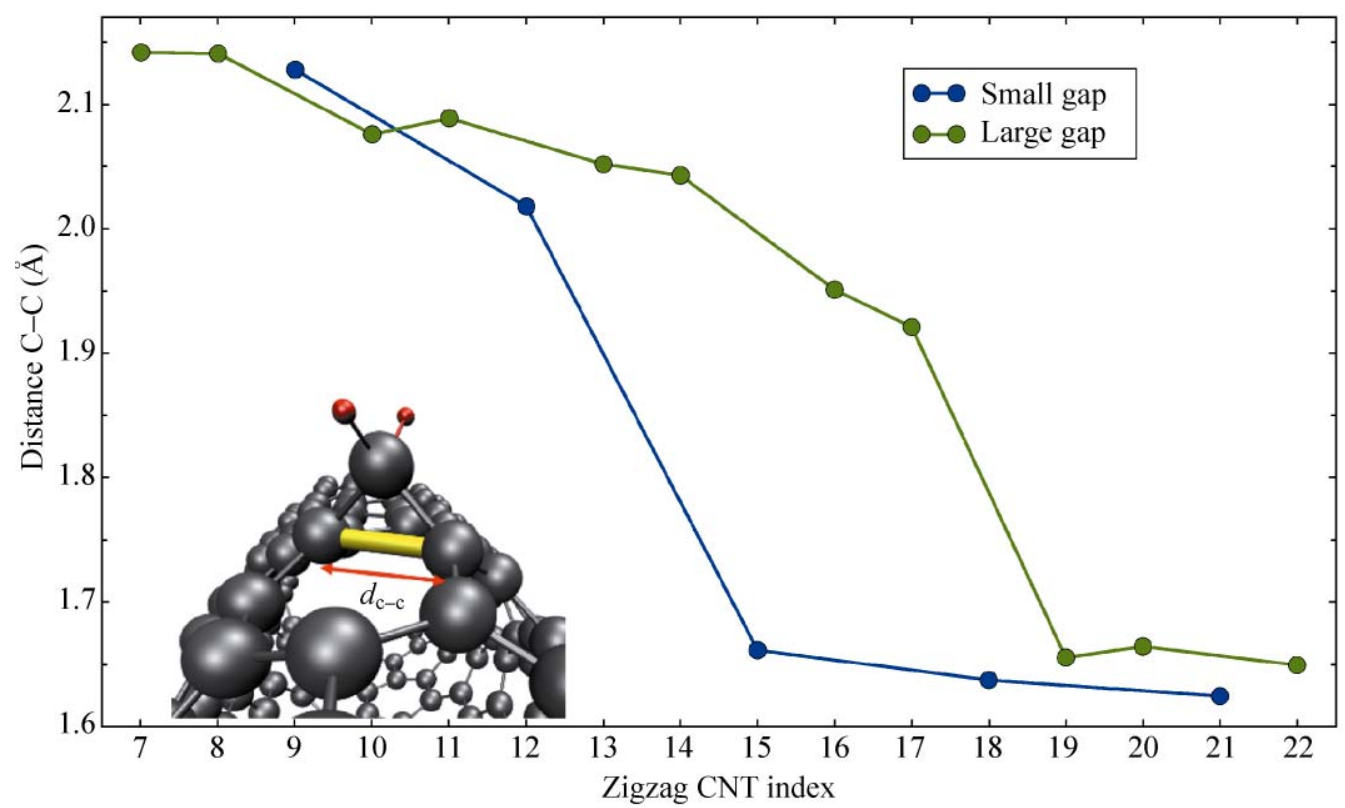

Figure 1 Main frame: sidewall equilibrium distances for the $\mathrm{C}-\mathrm{C}$ bond anchoring the carbene group, as a function of the tube $(n, 0)$ index, for both types of zigzag nanotubes: small gap $(n=3 m)$ and large gap $(n=3 m \pm 1)$ CNTs, with $n, m$ integer numbers and $(n, m)$ indicating the CNT chirality in the two-dimensional honeycomb lattice. A ball-and-stick model illustrates the $(1)$ skewed bonding configuration 
have been outlined (yellow links). This is at odds with the case of metallic armchair tubes for which (a) the parallel configuration does not exist, and (b) bonds perpendicular to the tube axis offer preferential addition sites [20]. In each case, the equilibrium position is reached when the carbon atom of $\mathrm{CH}_{2}$ is located on top of the $\mathrm{C}-\mathrm{C}$ tube bond forming a bridge-like structure [14, 20, 21, 25]. Our calculations shows that the $(\backslash)$ conformation is always more stable than the (II) one. This is consistent with the result of Chen et al. [19] for the case of a small $(8,0)$ finite-size cluster. It is further consistent with the study by Lee and Marzari on metallic armchair tubes showing that bonds orthogonal to the tube axis are preferentially grafted $[20,25]$. As a rule of thumb summarizing all these results, bonds with largest angle with respect to the tube axis are more active towards adsorption, since they experience most of the tube curvature. We therefore focus on the skewed configurations and show in Fig. 1 the evolutions of the sidewall bond distance $\left(d_{\mathrm{C}-\mathrm{C}}\right)$ between the two carbon atoms anchoring the carbene group as a function of zigzag CNT index, for both large and small gap CNTs [26].

As observed in the case of armchair tubes for the most stable orthogonal configuration [20, 25], there is a transition from an "open-skewed" configuration at small diameter, with $d_{\mathrm{C}-\mathrm{C}}=2.1 \AA$ for the $(8,0) \mathrm{CNT}$, to a closed one, with $d_{\mathrm{C}-\mathrm{C}}=1.65 \AA$ for the $(22,0)$ tube. The transition occurs for the $(15,0)$ tube with a diameter of $11.7 \AA$, at much smaller a diameter than for armchair tubes for which the transition occurs for the $(18,18)$ tube $($ diameter $=24.5 \AA)$. Such results can be rationalized by remarking that bonds along the circumferential direction undergo the largest tensile stress induced by curvature and are thus more prone to open. As a matter of fact, we find that bonds parallel to the tube axis, which do not feel the effect of curvature, always exhibit the closed configuration. An additional interesting observation is that large gap zigzag tubes exhibit a transition to the closed configuration at a larger diameter than small gap ones. This transition to closed configurations at rather small diameters for semiconducting tubes bears important consequences on electronic transport, as explained hereafter.

\section{Quantum electronic transport in func- tionalized nanotubes}

To explore mesoscopic electronic transport in long and disordered nanotubes, we follow a well-established computational strategy as discussed in prior works [27-33]. From the first-principles calculations for long tube sections functionalized by single groups, $a b$ initio Hamiltonian and overlap matrices are obtained. The atomic-like features of the basis set utilized by SIESTA allow us to have a relatively small and manageable description of the interaction between atoms with sparse Hamiltonian matrices. Such a set of initial building blocks is then used to construct micrometer long nanotubes composed by a random series of functionalized and pristine tube sections, introducing rotational and translational disorders. Functionalized CNT unit cells are long enough to avoid spurious interactions between virtual images during DFT calculations, so that slab-extremes are converged to the clean system. Functionalized and clean sections of CNTs can then be matched to build up long systems with perfect contact areas between the building blocks [34].

Standard techniques to calculate the Green's function associated with the sparse Hamiltonian and overlap matrices are further employed to include recursively the contribution of additional sections, leading to an $\mathrm{O}(\mathrm{N})$ scheme with respect to the tube length. Firstprinciples transport calculations of complex and disordered systems with several thousands of atoms can therefore be achieved. Assuming that the total system is made by a functionalized nanotube connected to two semi-infinite perfect CNTs leads, the conductance can be computed using the LandauerBüttiker transport theory $[12,13]$.

We first discuss the effect of a single functional group and show in Fig. 2 the energy-resolved conductance for a $(19,0)$ CNT (main frame) and a $(12,0)$ CNT (inset). These tubes are on both sides of the "open-to-closed" $\mathrm{C}-\mathrm{C}$ bond distance transition for the skewed $(\backslash)$ configuration (see Fig. 1). In the case of the small gap $(12,0) \mathrm{CNT}$, the grafted functional group in the $(\backslash)$ configuration, with open $\mathrm{C}-\mathrm{C}$ bond, has hardly any effect on the conductance as shown in the inset (blue curve). On the contrary, the parallel (II) bonding 


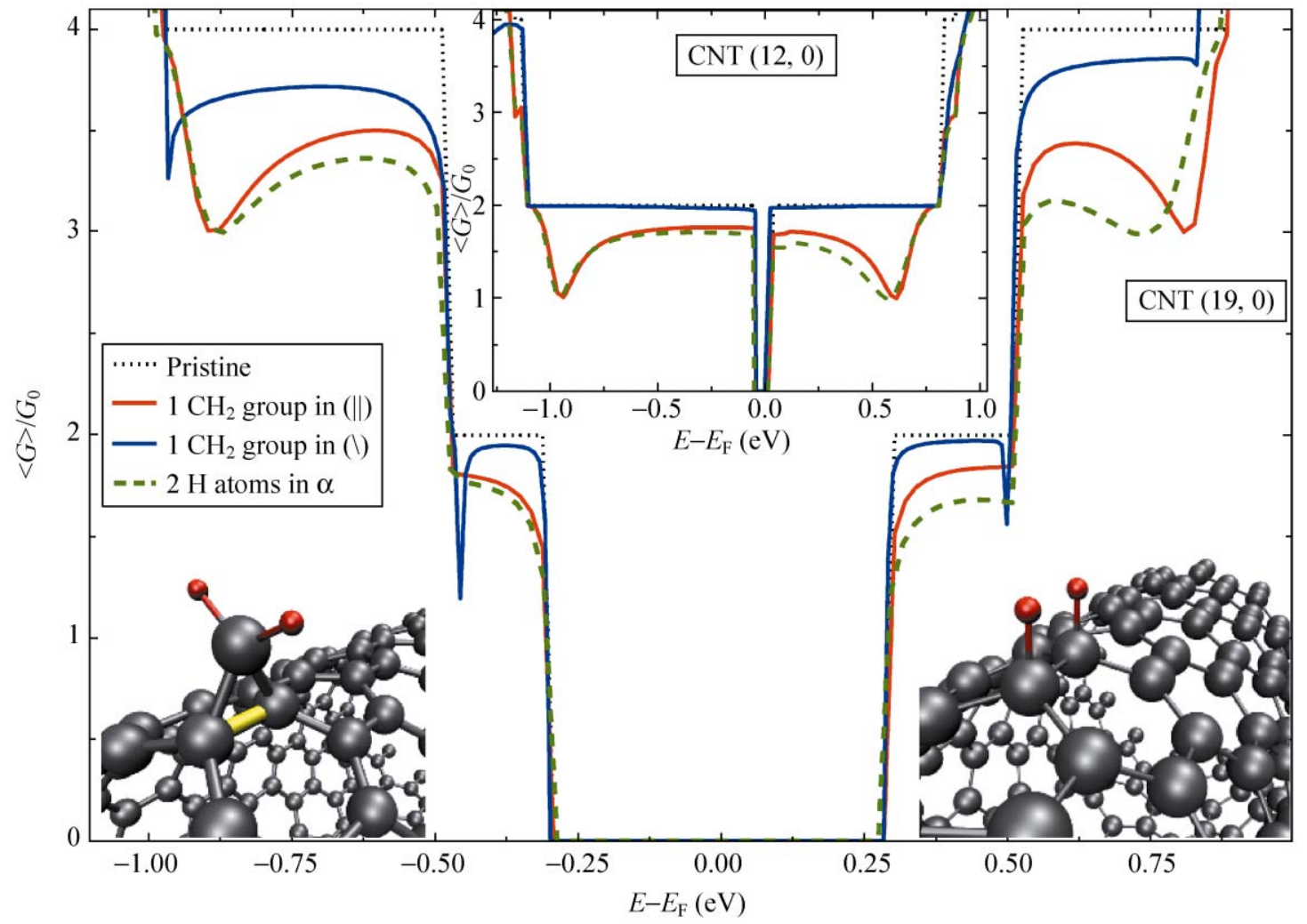

Figure 2 Main frame: conductance (in $G_{0}=2 \mathrm{e}^{2} / \mathrm{h}$ unit) of a pristine $(19,0)$ nanotube (dotted line) and one with a carbene group grafted in the parallel $(\|)$ (red line) and skewed $(\backslash)$ (blue line) configurations. The carbene functional group in the $\|$ conformation shows similar conductance profile to two grafted hydrogen atoms (green dashed line). Inset: the same as in the main frame but for a small gap $(12,0)$ nanotube. Ball-and-stick models of the $(\|)$ configuration and dihydrogenation are also given in the insets for illustration

geometry, with closed C-C bond, yields a more significant drop of conductance at nearly all energies (red curve inset). Such a contrasting behavior can be rationalized by observing that in the open geometry, the carbon atoms remain three-fold coordinated preserving thus the conjugated character of the $\mathrm{sp}^{2}$ pristine nanotube network [14, 20,21]. In contrast, in the closed geometry, the bridged carbon atoms are four-fold coordinated, destroying the local conjugated character of the $\pi$-network and thus reinforcing backscattering efficiency [12-14]. Such a transition to a local $\mathrm{sp}^{3}$ character can be strikingly evidenced by comparing our data with the conductance profile generated by two hydrogen atoms bonded to neighboring $\mathrm{C}$ atoms on a (II) bond (see Fig. 2). Clearly, the two kinds of functionalization lead to very similar conductance profiles with up to one full conducting channel quenched at selected energies on the first plateaus on each side of the gap.
Assuming that all addends will be grafted in the skewed configuration, one may conclude that the conductance of the small gap $(12,0)$ tube can be preserved up to very large coverages. For a $(10,10)$ armchair tubes, it was shown that up to several hundreds of carbene groups could be grafted while preserving nearly $75 \%$ of the conductance at all energies [14]. However, as shown above, the open-to-closed transition in zigzag tubes occurs at much smaller diameter as compared to armchair tubes, so that basically all zigzag tubes with a diameter larger than 1.2-1.5 nm will exhibit closed grafting configurations, even for the most stable skewed bonds. As a result, and as shown in Fig. 2 for the $(19,0)$ tube, even an isolated carbene group grafted on a skewed bond induces substantial backscattering close to band edges (onsets of plateaus). Even though the effect is less pronounced than that induced by a carbene group or two hydrogen atoms on a parallel bond (red and

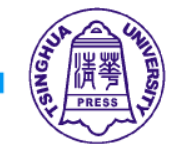

Springer 
dashed green lines), the consequences become rapidly dramatic even for a few tens of attached functional groups, as discussed hereafter.

To further explore the electronic transport properties of long and chemically modified semiconducting nanotubes, we have performed a mesoscopic study for several tubes with a length of $1 \mu \mathrm{m}$ and random grafting of an increasing number of carbene groups. Conductances were averaged over up to 20 configurations of disorder for a given tube and number of functional groups. As a representative case, Fig. 3 shows a comparison between the $(10,0)$ and the $(22,0)$ tubes for a single grafted carbene group and for an increasing number of groups $(25,50$, and 100$)$ in the ( $\backslash$ ) conformation. For a single grafted group, we revisit the results of Fig. 2 established for the $(12,0)$ and $(19,0)$ tubes: the backscattering is negligible for very small tubes, but becomes significant for tubes such as the $(22,0)$ with a diameter beyond the openclosed bond transition value $(\sim 1.2-1.4 \mathrm{~nm})$. This initial difference is then amplified when adding a much larger number of addends, which enhances disorder effects and allows quantum interferences between scattering centers to set in. As a result, for the same number of grafted groups, their impact on electronic transport in both semiconducting tubes becomes markedly different. While the $(10,0)$ manifests a weak reduction of conductance on the first plateau (which remains close to $2 G_{0}$ even for the largest number of addends), a strong decay is observed for the $(22,0)$ with an almost fully suppressed conductance even for the lower studied density (25 groups randomly distributed over $1 \mu \mathrm{m}$ ).

To characterize the related conduction regimes, we report in Fig. 4, the average conductance for $(12,0)$ (left frame) and $(19,0)$ (right frame) $\mathrm{CH}_{2}$-functionalized CNTs as a function of the number of attached carbene groups. Both $(\backslash)$ and (II) bonding configurations of $\mathrm{CH}_{2}$ are considered, together with two hydrogen atoms in the parallel configuration (see above). The average is performed over 200 configurations of disorder and at the selected energy $E-E_{\mathrm{F}}=0.35 \mathrm{eV}$, that is roughly

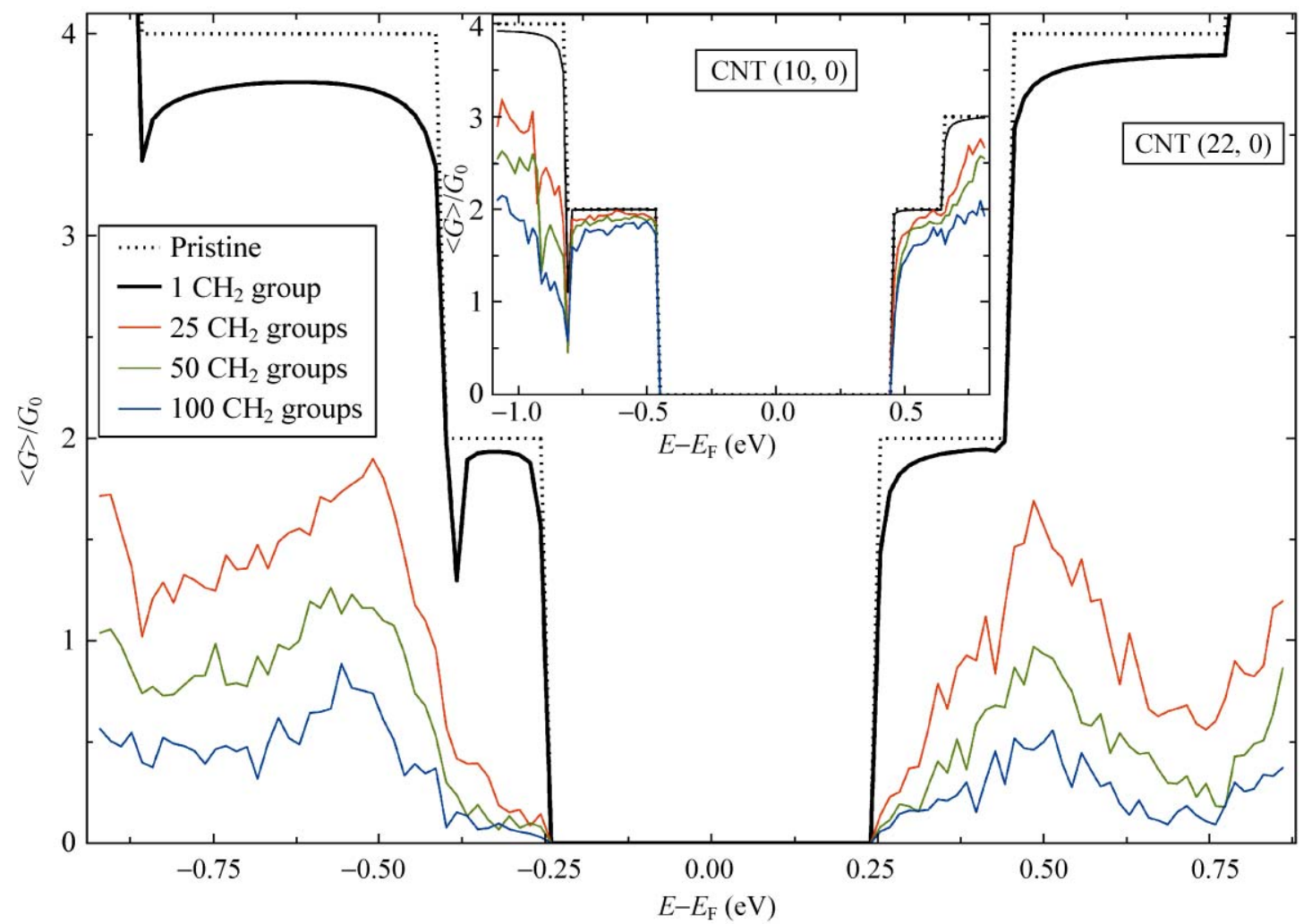

Figure 3 Main frame: conductance for a pristine $(22,0)$ nanotube (dotted line), with a single grafted carbene group in the $(\backslash)$ conformation (solid line), and for averaged configurations with varying addend densities (25, 50 or $100 \mathrm{CH}_{2}$ groups distributed at random along one $\left.\mu \mathrm{m}\right)$. Inset: the same as in the main frame but for the smaller diameter $(10,0)$ semiconducting nanotube 

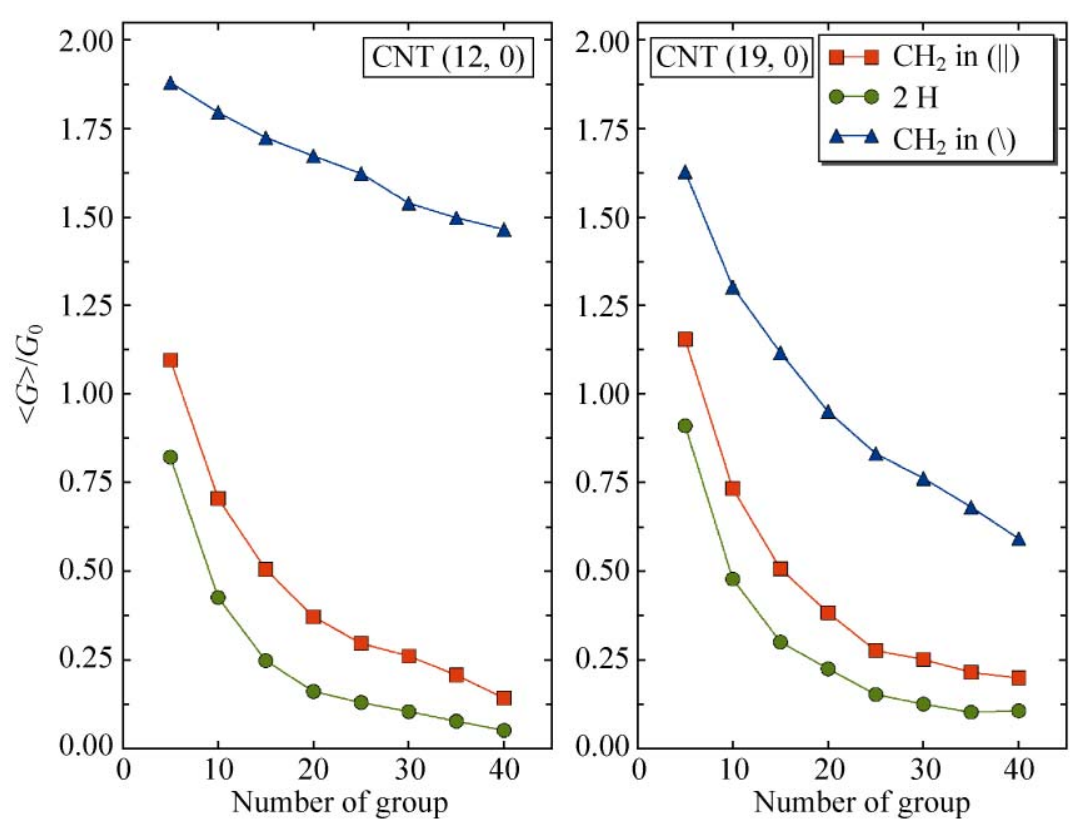

Figure 4 Plots of average conductance versus number of attached carbene groups for the $(12,0)$ and $(19,0)$ nanotubes, for $\mathrm{CH}_{2}$ addends in different conformations and for dihydrogenation

at the center of the first electron sub-band. For the $(\backslash)$ bonding in the $(12,0)$ tube, a weak conductance decay evidences a quasi-ballistic regime for the considered addends density. In contrast, for the (II) bonding configuration, the conductance decrease is roughly inversely proportional to the number of grafted groups, indicating a diffusive regime. This results in a conductance reduction by one order of magnitude for only 40 groups distributed over $1 \mu \mathrm{m}$, showing the large impact of such bonding type. The increase in diameter further degrades the conductance properties. In the $(19,0)$ case, even the more favorable $(\backslash)$ bonding leads now to a quick drop of conductance with increasing number of grafted groups.

The early transition from the open to closed configuration for carbene groups grafted onto zigzag nanotubes, with a critical diameter of about $1.2 \mathrm{~nm}$, represents a serious drawback for the use of such functional groups in the case of semiconducting nanotubes. While smaller diameter tubes can always be synthesized, mass produced CNTs exhibit a mean diameter which does not go below such a critical value. As demonstrated above, for standard semiconducting tubes, carbene functionalization is thus expected to lead to a drastic reduction of conductance with only a few functional groups. Besides, it was shown that carbene group desorbs easily at room temperature from graphene, that is in the closed $\mathrm{C}-\mathrm{C}$ bond configuration [35]. Namely, the desorption barrier (a few tenths of $1 \mathrm{eV}$ ) can be easily overcome in ambient conditions. It is therefore not even clear that, beyond the transition diameter, carbene grafting can be a viable way for functionalizing zigzag nanotubes.

\section{Conclusions}

By using a fully $a b$ initio transport approach, we have explored the electronic transport regimes in chemically functionalized semiconducting carbon nanotubes, assessing the true potential of cycloaddition reactions as a precursor for further enhancement of devices functionalities. Small diameter functionalized tubes have been shown to preserve good conduction ability (as for the case of weak physisorption [36]), whereas a strongly diffusive regime was obtained for larger diameter tubes, similar to the effect of a low dihydrogenation density. This effect was explained by the orbital rehybridization of the atoms to which functional groups are attached.

One should note that our transport methodology neglects many important physical effects that will take place in an out-of-equilibrium situation. In particular,

\section{(1) Springer}


electron-phonon scattering, impurity screening, and non-equilibrium charge redistribution should be included and treated self-consistently to allow for a more quantitative comparison with experiments. This is beyond the scope of our present study, but should be considered in future works.

Our study quantifies the severe limits of divalent additions in large diameter semiconducting nanotubes, and suggests that nanotubes with smaller diameter sizes should be targeted to engineer efficient novel device functionalities.

\section{Acknowledgements}

We thank the Centre de Calcul Recherche et Technologie of the Commissariat à l'Energie Atomique supercomputing facilities for providing computational resources and technical support. Financial support from the Agence National pour la Recherche (programme ANR/PNANO) project ACCENT is acknowledged. A. L. B. acknowledges support from the Marie-Curie fellowship CHEMTRONICS program. S. R. is indebted to the Alexander von Humboldt Foundation for financial support. Francois Triozon, Luigi Genovese, and Thierry Deutsch are acknowledged for fruitful discussions.

Open Access: This article is distributed under the terms of the Creative Commons Attribution Noncommercial License which permits any noncommercial use, distribution, and reproduction in any medium, provided the original author(s) and source are credited.

\section{References}

[1] Charlier, J. C.; Blase, X.; Roche, S. Electronic and transport properties of nanotubes. Rev. Mod. Phys. 2007, 79, 677-732.

[2] Guo, X. F.; Huang, L. M.; O’Brien, S.; Kim, P.; Nuckolls, K. Directing and sensing changes in molecular conformation on individual carbon nanotube field effect transistors. $J$. Am. Chem. Soc. 2007, 127, 15045-15047.

[3] Simmons, J. M.; In, I.; Campbell, V. E.; Mark, T. J.; Léonard, F.; Gopalan, P.; Eriksson, M. A. Optically modulated conduction in chromophore-functionalized single-wall carbon nanotubes. Phys. Rev. Lett. 2007, 98, 086802.

[4] Borghetti, J.; Derycke, V.; Lenfant, S.; Chenevier, P.; Filoramo, A.; Goffman, M.; Vuillaume, D.; Bourgoin, J. P.
Optoelectronic switch and memory devices based on polymer-functionalized carbon nanotube transistors. $A d v$. Mater. 2006, 18, 2535-2540.

[5] Mannik, J.; Goldsmith, B. R.; Kane, A.; Collins, P. G. Chemically induced conductance switching in carbon nanotube circuits. Phys. Rev. Lett. 2006, 97, 016601.

[6] Goldsmith, B. R.; Coroneus, J. G.; Khalap, V. R.; Kane, A. A.; Weiss, G. A.; Collins, P. G. Conductance-controlled point functionalization of single-walled carbon nanotubes. Science 2007, 315, 77-81.

[7] Meunier, V.; Kalinin, S. V.; Sumpter, B. G. Nonvolatile memory elements based on the intercalation of organic molecules inside carbon nanotubes. Phys. Rev. Lett. 2007, 98, 056401.

[8] Derycke, V.; Auvray, S.; Borghetti, J.; Chung, C. L.; Lefèvre, R.; Lopez-Bezanilla, A.; Nguyen, K.; Robert, G.; Schmidt, G.; Anghel, C.; Chimot, N.; Lyonnais, S.; Streiff, S.; Campidelli, S.; Chenevier, P.; Filoramo, A.; Goffman, M. F.; Goux-Capes, L.; Latil, S.; Blase, X.; Triozon, F.; Roche S.; Bourgoin, J. P. Carbon nanotube chemistry and assembly for electronic devices. C. R. Physique 2009, 10, 330-347.

[9] Bahr, J. L.; Tour, J. M. Highly functionalized carbon nanotubes using in situ generated diazonium compounds. Chem. Mater. 2001, 13, 3823-3824.

[10] Cabana, J.; Martel, R. Probing the reversibility of sidewall functionalization using carbon nanotube transistors. J. Am. Chem. Soc. 2007, 129, 2244-2245.

[11] Balasubramanian, K.; Lee, E. J. H.; Weitz, R. T.; Burghard, T.; Kern, T. Carbon nanotube transistors-chemical functionalization and device characterization. Phys. Status Solidi A 2008, 205, 633-646.

[12] Le, Y. S.; Nardelli, M. B.; Marzari, N. Band structure and quantum conductance of nanostructures from maximally localized wannier functions: The case of functionalized carbon nanotubes Phys. Rev. Lett. 2005, 95, 076804.

[13] Triozon, F.; Lambin, P.; Roche, S. Electronic transport properties of carbon nanotube based metal/semiconductor/metal intramolecular junctions. Nanotechnology 2005, 16, 230-233.

[14] Lopez-Bezanilla, A.; Triozon, F.; Latil, S.; Blase, X.; Roche, S. Effect of the chemical functionalization on charge transport in carbon nanotubes at the mesoscopic scale. Nano Lett. 2009, 9, 940-944.

[15] Chen, Y.; Haddon, R. C.; Fang, S.; Rao, A. M.; Lee, W. H.; Dickey, E. C.; Grulke, E. A.; Pendergrass, J. C.; Chavan, A.; Haley, B. E.; Smalley, R. E. Chemical attachment of organic functional groups to single-walled carbon nanotube material. J. Mater. Res. 1998, 13, 2423-2431.

[16] Chen, J.; Hamon, M. A.; Hu, H.; Chen, Y. S.; Rao, A. M.; 
Eklund, P. C.; Haddon, R. C. Solution properties of singlewalled carbon nanotubes Science 1998, 282, 95-98.

[17] Holzinger, M.; Vostrowsky, Q.; Hirsch, A.; Hennrich, F.; Kappes, M.; Weiss, R.; Jellen, F. Sidewall functionalization of carbon nanotubes. Angew. Chem. Int. Ed. 2001, 40, 4002-4005.

[18] Coleman, K. S.; Bailey, S. R.; Fogden, S.; Green, M. L. H. Functionalization of single-walled carbon nanotubes via the bingel reaction. J. Am. Chem. Soc. 2003, 125, 8722-8723.

[19] Chen, Z. F.; Nagase, S.; Hirsch, A.; Haddon, R. C.; Thiel, W.; Schley, P. C. Side-wall opening of single-walled carbon nanotubes (SWCNTs) by chemical modification: A critical theoretical study. Angew. Chem. Int. Ed. 2004, 43, 1552-1554.

[20] Lee, Y. S.; Marzari, N. Cycloaddition functionalizations to preserve or control the conductance of carbon nanotubes. Phys. Rev. Lett. 2006, 97, 116801.

[21] Park, H.; Zhao, J. J.; Lu, J. P. Effects of sidewall functionalization on conducting properties of single wall carbon nanotubes. Nano Lett. 2006, 6, 916-919.

[22] Sánchez-Portal, D.; Ordejon, P.; Artacho, E.; Soler, J. M. Density-functional method for very large systems with LCAO basis sets. Int. J. Quant. Chem. 1997, 65, 453-461.

[23] Soler, J. M.; Artacho, E.; Gale, J. D.; Garcia, A.; Junquera, J.; Ordejon, P.; Sanchez-Portal, D. The SIESTA method for $a b$ initio order- $N$ materials simulation. J. Phys.: Condens. Matter 2002, 14, 2745-2779.

[24] Troullier, N.; Martins, J. L. Efficient pseudopotentials for plane-wave calculations. Phys. Rev. B 1991, 43, 1993-2006.

[25] Lee, Y. S.; Marzari, N. Cycloadditions to control bond breaking in naphthalenes, fullerenes, and carbon nanotubes: A first-principles study. J. Phys. Chem. C 2008, 112, 4480-4485.

[26] Kane, C. L.; Mele, E. L. Size, shape, and low energy electronic structure of carbon nanotubes. Phys. Rev. Lett. 1997, 78, 1932.
[27] Biel, B.; García-Vidal, F. J.; Rubio, A.; Flores, F. Ab initio study of transport properties in defected carbon nanotubes: An O(N) approach. J. Phys: Condes. Matter 2008, 20, 294214.

[28] Adessi, Ch.; Roche, S.; Blase, X. Reduced backscattering in potassium-doped nanotubes: $A b$ initio and semiempirical simulations. Phys. Rev. B 2006, 73, 125414.

[29] Triozon, F.; Roche, S. Efficient linear scaling method for computing the Landauer-Büttiker conductance. Eur. Phys. J. B 2005, 46, 427-431.

[30] Triozon, F.; Lambin, P.; Roche, S. Electronic transport properties of carbon nanotube based metal/semiconductor/metal intramolecular junctions. Nanotechnology 2005, 16, 230-233.

[31] Avriller, R.; Roche, S.; Triozon, F.; Blase, X. Lowdimensional quantum transport properties of chemicallydisordered carbon nanotubes: From weak to strong localization regimes. Mod. Phys. Lett. B 2007, 21, 1955-1982.

[32] Rocha, A. R.; Rossil, M.; Fazzio, A.; da Silva, A. J. R. Designing real nanotube-based gas sensors. Phys. Rev. Lett. 2008, 100, 176803.

[33] Markussen, T.; Rurali, R.; Brandbyge, M.; Jauho, A. P. Electronic transport through Si nanowires: Role of bulk and surface disorder. Phys. Rev. B 2006, 74, 245313.

[34] The convergence to the clean system at the boundaries of a functionalized nanotube section can be checked through comparison of the conductance profiles obtained from its last layers (dotted lines in Fig. 2 and Fig. 3) with those of pristine nanotubes sections.

[35] Margine, E. R.; Bocquet, M. L.; Blase, X. Thermal stability of graphene and nanotube covalent functionalization. Nano Lett. 2008, 8, 3315-3319.

[36] Latil, S.; Roche, S.; Charlier, J. C. Electronic transport in carbon nanotubes with random coverage of physisorbed molecules. Nano Lett. 2005, 5, 2216-2219. 